Journal of the Operations Research

Society of Japan

Vol. 30, No. 4, December 1987

\title{
THE GENERALIZED LINEAR SEARCH PROBLEM, EXISTENCE OF OPTIMAL SEARCH PATHS
}

\author{
Zaid T. Balkhi
}

King Saud University

(Received March 19, 1986; Revised December 15, 1986)

\begin{abstract}
A target $\mathrm{x}$ is a point on the real line given by the value of a random variable $\mathrm{X}$, which has some distribution function F. A searcher starts looking for $\mathrm{x}$ from some point on the line, using a continuous path. He makes for $\mathrm{x}$ with un upper bound on his speed till he finds it. The target being sought for might be in either direction from the starting point, so the searcher has in general to retrace his steps many times before he attains his goal. It is desired to search in an optimal manner so as to minimize the expected cost of the search. All previous papers treated this problem using the origin as the starting point of the search. They have been proved that one can minimize the expected cost if the underlying distribution satisfies certain conditions. In this paper, the problem will be treated in the "General Case", which means that the search may start from any point on the real line. Conditions under which we can minimize the expected cost in the general case will be given.
\end{abstract}

\section{Introduction}

The linear search problem concerns with searching for a hidden target $x$ on the real line $\mathbb{R}$. The position of the target is given by the value of a random variable $X$, which has a known or unknown distribution function $F$. A searcher starts looking for the target at some point $a_{0}\left(\left|a_{0}\right|<\infty\right)$. He moves continuously along the line in both directions of the starting point $a_{0}$ until the target is located. The searcher would change his direction, at suitable points, many times before attaining his goal. Thus we might consider the path length as the cost of the search. It is the aim of the searcher to minimize this expected cost. Authors in [5], [6], [7], [8], [10], [11] and [13] have considered only sequential search paths (S.S.P) with $a_{0}=0$. We shall first give a review of their results: 
Define $\quad c=\inf \{t: F(t)>0\}, \quad d=\sup \{t: F(t)<1\}$,

A (S.S.P), then can be represented as a sequence $a=\left\{a_{i} ; i \geq 0\right\}$ such that, either

$$
\cdots a_{5} \leq a_{3} \leq a_{1} \leq a_{0}=0 \leq a_{2} \leq a_{4} \leq a_{6} \leq \cdots
$$

with $a_{2 i-1} \rightarrow c$ and $a_{2 i} \rightarrow d_{\text {, or }}$

$$
\cdots \leq a_{6} \leq a_{4} \leq a_{2} \leq a_{0}=0 \leq a_{1} \leq a_{3} \leq a_{5} \leq \cdots
$$

with $a_{2 i-1} \rightarrow d$ and $a_{2 i} \rightarrow c$. When all inequalities are strict, the search path is said to be strong, otherwise it is said to be weak. Denote by $Q_{0}$ the class of all such search paths, and by $D(a, x)$ the total distance travelled from the starting point to $x$, using the search path $a=\left\{a_{i} ; i \geq 0\right\}$. Then $D(a, x)$ is a random variable and we denote by $D(a, F)$ its expected value. As a notational convenience let $a_{-1}=0$, then

$$
\text { (1.3) } \quad D(a, x)=|x|+2 \sum_{i=1}^{n-1}\left|a_{i}\right|
$$

Where $x$ lies between $a_{n-2}$ and $a_{n} ; n=1,2,3, \ldots$. As further notational convenience we shall use $\int_{t_{1}}^{t} 2 d F(t)$ in place of $\left|\int_{t_{1}}^{t} 2 d F(t)\right|$ regardless of the order of $t_{1}$ and $t_{2}$. The expected cost is then given by

$$
D(a, F)=A(F)+\Delta_{0}(a, F)
$$

where

$$
A(F)=\int_{C}^{d}|x| d F(x) \text { is the first absolute moment of } F \text {. }
$$

$$
\begin{aligned}
\Delta_{0}(a, F) & =2 \sum_{n=1}^{\infty} \sum_{i=1}^{n-1}\left|a_{i}\right| \int_{a_{n-2}}^{a} d F \\
& \left.=2 \sum_{n=1}^{\infty}\left|a_{n}\right| \underset{a_{n-1}}{\left(1-\int_{n}^{a} n\right.} d F\right)
\end{aligned}
$$

(see, [1], [5], [10] and [11]). Thus, our aim is to minimize $D(a, F)$ (or equivalent $1 \mathrm{y} \Delta_{0}(a, F)$ for fixed $\left.F\right)$. Define

$$
m_{0}=\inf \left\{D(a, F): a \varepsilon Q_{0}\right\}
$$

then the main problem is to find a search path $a=\left\{a_{i} ; i \geq 0\right\}$ from class $\ell_{0}$ 
such that $D(a, F)=m_{0}$. If such a search path exists, we call it an optimal search path (O.S.P).

The existence of optimal search paths in class $\ell_{0}$ has been established by many authors assuming that the underlying distribution satisfies certain conditions. Franck [10] proved the following result (see also [1]).

Theorem 1.1: There exists a search path from class $Q_{0}$ with finite expected cost if and only if $A(F)<\infty$.

Thus, whenever $A(F)<\infty$, then the infimum $m_{0}<\infty$ is guaranteed. However, some more conditions are to be imposed on $F$ in order to attain such infimum. Franck [10] imposed the following condition :

"There exists a nondegenerate interval $[a, b]$ with $0 \varepsilon[a, b]$ and a constant $k>0$ such that, for distinct $c_{1}, c_{2} \varepsilon[a, b]$ we have

$$
\left[F\left(c_{1}\right)-F\left(c_{2}\right)\right] /\left(c_{1}-c_{2}\right) \leq k^{\prime \prime} \text {. }
$$

Beck [5], however, imposed the condition that:

"At leas one of

$$
\begin{aligned}
& F^{-}(0)=\lim _{t \rightarrow 0^{-}} F(t)-F(0), \\
& F^{+}(0)=\lim _{t \rightarrow 0^{+}} F(t)
\end{aligned}
$$

is finite"

Both Franck [10] and Beck [5] proved the following theorem.

Theorem 1.2: If $A(F)<\infty$, then there exists an (O.S.P) from class $Q_{0}$ if and only if (1.9) ((1.8) in [10]) holds.

Fristedt and Heath [11] adopted more general approach. Under some assumptions they proved the following two theorems.

Theorem 1.3: If $A(F)<\infty$, then there exists an (O.S.P) which is sequential and has a constant speed equals 1 .

Theorem 1.4: If $A(F)<\infty$ then all optimal search paths are sequential. Thus, in view of the last two theorems, it is reasonable to restrict attention to sequential search paths for which the searcher's speed is equal to 1 . The expected cost, then, is either the expected path length $D(a, F)$, or the expected searching time $T(a, F)$ i.e.:

(*) Franck's condition and Beck's condition are not equivalent. One can easily show that (1.8) implies (1.9) but not conversely. 
$(1.10)$

$$
D(a, F)=T(a, F)=A(F)+\Delta_{0}(a, F)
$$

It is intuitively clear that one might shorten the travelled distance, hence the expected cost, if he starts the search from some point on the real line, other than the origin (see Balkhi [1], Table - 1 through Table - 6).

A new kind of search path in which the search may start from any point on the line has been shown by Balkhi [1]. The research of [1], in fact, has focussed on building the mathematical model for the expected cost for all possible cases of search, and on finding an algorithm for constructing minimal search paths at each possible case. A numerical solution, then has been found by means of computers considering, without proofs, that, there exists an optimal search path at each possible case. These numerical results, then confirm the claim that: These new kinds of search paths give less expected cost than the earlier one.

$$
* * *
$$

In this paper, we sha11 use techniques and arguments, similar to those used by Beck [5], to establish the existence of optimal search paths in the "Genera1 Case".

Let us first exclude some trivial cases. If $a_{0}=c$ (or $a_{0}=d$ ), the only reasonable way to search, then, is to start from the point $c$ (or d) moving to the right (or to the left) until the target is found. Using the above assumptions and all other assumptions mentioned in Beck [5], then, there are only five classes of possible search paths, one of which is class $Q_{0}$. The other four will be shown in section 2. Sufficient conditions under which there exists an (O.S.P) in each class will be developed in section 3. Some applications of linear search problems can be found in section 4.

\section{Search Paths in the General Case}

In each of the following four cases we have two dual search paths depending on whether $a_{0} \geq 0$ or $a_{0} \leq 0$ and for each search path, either $a_{2 i-1} \rightarrow c a_{2 i} \rightarrow d$, or vice versa.

Case (1) This case consists of all search paths such that, either

$$
\cdots a_{5} \leq a_{3} \leq a_{1} \leq 0 \leq a_{0} \leq a_{2} \leq a_{4} \leq a_{6} \leq \cdots
$$

or

$$
\cdots a_{6} \leq a_{4} \leq a_{2} \leq a_{0} \leq 0 \leq a_{1} \leq a_{3} \leq a_{5} \leq \ldots
$$


Case (2): In this case we have either

$$
\cdots a_{6} \leq a_{4} \leq a_{2} \leq 0 \leq a_{0} \leq a_{1} \leq a_{3} \leq a_{5} \leq \ldots
$$

or

$$
\cdots a_{5} \leq a_{3} \leq a_{1} \leq a_{0} \leq 0 \leq a_{2} \leq a_{4} \leq a_{6} \leq \ldots
$$

Case (3): Let $J$ be a "finite and nonempty set" of odd numbers. For i $\varepsilon_{J}$, we either take

$$
\cdots \leq a_{j+4} \leq a_{j+2} \leq 0 \leq a_{j} \leq a_{j-2} \leq \ldots \leq a_{1} \leq a_{0} \leq a_{2} \leq \ldots \leq a_{j-1} \leq a_{j+1} \leq \ldots
$$

or

$$
\cdots \leq a_{j+3} \leq a_{j+1} \leq a_{j-1} \leq \ldots \leq a_{2} \leq a_{0} \leq a_{1} \leq \ldots \leq a_{j-2} \leq a_{j} \leq 0 \leq a_{j+2} \leq a_{j+4} \leq \ldots
$$

Case (4): We consider, in this case, J to be a "finite and nonempty set" of even numbers. For $j \varepsilon J$ we either have

$$
\cdots \leq a_{j+4} \leq a_{j+2} \leq 0 \leq a_{j} \leq a_{j-2} \leq \ldots \leq a_{2} \leq a_{0} \leq a_{1} \leq a_{3} \leq \ldots \leq a_{j=1} \leq a_{j+1} \leq \ldots
$$

or

$$
\cdots_{j+3} a_{j+1} \leq a_{j-1} \leq \ldots \leq a_{1} \leq a_{0} \leq a_{2} \leq \ldots \leq a_{j-2} \leq a_{j} \leq 0 \leq a_{j+2} \leq a_{j+4} \leq \ldots
$$

Designate by $Q_{k}$ the class of all search paths in case $(k) ; k=0,1,2,3$ and 4 (The earlier case mentioned in section 1 will, therefore, be referred as case (0))

Theorem 2.1: If $a=\left\{a_{i} ; i \geq 0\right\} \varepsilon Q_{k} ; k=0,1,2,3$, and 4 , then the expected cost of the search is given by

$$
D_{k}(a, F)=T_{k}(a, F)=A(F)+\Delta_{k}(a, F) ; k=0,1,2,3 \text { and } 4
$$

where:

$$
\begin{gathered}
\Delta_{0}(a, F)=2 \sum_{i=1}^{\infty}\left|a_{i}\right|\left\{1-\operatorname{sign}\left(a_{i}\right)\left[F\left(a_{i}\right)-F\left(a_{i-1}\right)\right]\right\} \\
\Delta_{1}(a, F)=-2\left|\int_{0}^{a}\right| x|d F(x)|+\left|a_{0}\right|+2 \sum_{i=1}^{\infty}\left|a_{i}\right| x \\
\left\{1-\operatorname{sign}\left(a_{i}\right)\left[F\left(a_{i}\right)-F\left(a_{i-1}\right)\right]\right\}
\end{gathered}
$$

$$
\begin{gathered}
\Delta_{2}(a, F)=-2\left|\int_{0}^{a} 0\right| x|d F(x)|-\left|a_{0}\right|+2 \sum_{i=1}^{\infty}\left|a_{i}\right| x \\
\left\{1-\operatorname{sign}\left(a_{i}\right)\left[F\left(a_{i}\right)-F\left(a_{i-1}\right)\right]\right\}
\end{gathered}
$$




$$
\begin{aligned}
\Delta_{3}(a, F)=-2 \mid \int_{0}^{a} 0 & |x| d F(x)|+| a_{0}|-2| a_{j} \mid\left\{1+\operatorname{sign}\left(a_{0}\right)\left[F\left(a_{j}\right)-F\left(a_{0}\right)\right]\right\}+ \\
& 2 \sum_{i=1}^{(j-1) / 2}\left(\left|a_{2 i}\right|-\left|a_{2 i-1}\right|\right)\left\{1+\operatorname{sign}\left(a_{0}\right)\left[F\left(a_{1}\right)-F\left(a_{0}\right)\right]\right\}+ \\
& 2 \sum_{i=j+1}^{\infty}\left|a_{i}\right|\left\{1-\operatorname{sign}\left(a_{i}\right)\left[F\left(a_{i}\right)-F\left(a_{i-1}\right)\right]\right\}
\end{aligned}
$$

$$
\begin{aligned}
\Delta_{4}(a, F)=-2 \mid \int_{0}^{a} 0 & |x| d F(x)|-| a_{0}\left|-2 \operatorname{sign}\left(a_{0}\right) \cdot\right| a_{j} \mid\left[F\left(a_{j}\right)-F\left(a_{0}\right)\right]+ \\
& 2 \sum_{i=1}^{j / 2}\left(\left|a_{2 i-1}\right|-\left|a_{2 i}\right|\right)\left\{1-\operatorname{sign}\left(a_{0}\right)\left[F\left(a_{1}\right)-F\left(a_{0}\right)\right]\right\}+ \\
& 2 \sum_{i=j+1}^{\infty}\left|a_{i}\right|\left\{1-\operatorname{sign}\left(a_{i}\right)\left[F\left(a_{i}\right)-F\left(a_{i-1}\right)\right]\right\}
\end{aligned}
$$

Proof: Let $a=\left\{a_{i} ; i \geq 0\right\} \varepsilon Q_{k}$. The proof for $k=0$ is apparent, because we need only to discuss the signs of $a_{i} ; i \geq 1$ (recall that $a_{0}=0$ for this case). We shall give the proof, in detail, only in one case, say $k=3$. The proof for the other cases can easily be given in the same way. The only possible locations of $x$, for $k=3$, are (see Figure - 1).

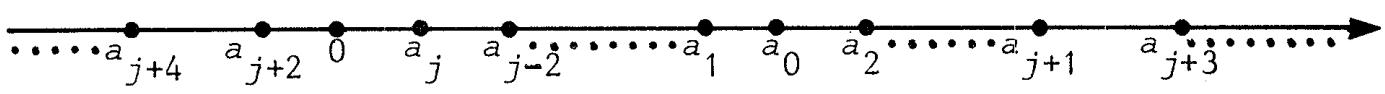

$$
\text { Figure - } 1
$$

$$
x \text { lies between } a_{n-2} \text { and } a_{n} \text { for } n \geq j+3 \text {, then }
$$

$$
D_{3}(a, x)=|x|+\left|a_{0}\right|+2 \sum_{i=1}^{(j-1) / 2}\left(\left|a_{2 i}\right|-\left|a_{2 i-1}\right|\right)-2\left|a_{j}\right|+2 \sum_{i=j+1}^{n-1}\left|a_{i}\right|
$$

$x$ lies between $a_{n-2}$ and $a_{n}$ for $n=2,4, \ldots, j+1$, then

$$
D_{3}(a, x)=|x|+\left|a_{0}\right|+2 \sum_{i=1}^{(j-1) / 2}\left(\left|a_{2 i}\right|-\left|a_{2 i-1}\right|\right)-2\left|a_{j}\right|
$$

$\quad x$ lies between $a_{n-2}$ and $a_{n}$ for $n=3,5, \ldots, j$, then

$$
D_{3}(a, x)=-|x|+\left|a_{0}\right|+2 \sum_{i=1}^{(j-1) / 2}\left(\left|a_{2 i}\right|-\left|a_{2 i-1}\right|\right)
$$

$$
x \text { is between } a_{0} \text { and } a_{1} \text {, then } D_{3}(a, x)=-|x|+\left|a_{0}\right|
$$


$\left(5^{\circ}\right)$

$$
x \text { is between } 0 \text { and } a_{j} \text {, then }
$$

$$
D_{3}(a, x)=-|x|+\left|a_{0}\right|+2 \sum_{i-1}^{(j-1) / 2}\left(\left|a_{2 i}\right|-\left|a_{2 i-1}\right|\right)-2\left|a_{j}\right|+2\left|a_{j+1}\right|
$$

$\left(6^{\circ}\right)$

$$
\begin{aligned}
& x \text { is between } 0 \text { and } a_{j+2} \text {, then } \\
& D_{3}(a, x)=|x|+\left|a_{0}\right|+2 \sum_{i=1}^{(j-1) / 2}\left(\left|a_{2 i}\right|-\left|a_{2 i-1}\right|\right)-2\left|a_{j}\right|+2\left|a_{j+1}\right|
\end{aligned}
$$

It is to be noted that the search covers the whole interval $[c, d]$. Therefore, we find:

$$
\begin{aligned}
& T_{3}(a, F)=D_{3}(a, F)=\left\{| a _ { 0 } | \left(\sum_{i=j+3}^{\infty} \int_{a_{i-2}}^{a_{i}} d F(x)+\int_{a_{0}}^{a_{j+1}} d F(x)+\int_{a_{1}}^{a_{j}} d F(x)+\int_{a_{0}}^{a_{1}} d F(x)+\right.\right. \\
& \left.\left.\int_{0}^{a_{j}} d F(x)+\int_{0}^{a_{j+2}} d F(x)\right)\right\}+\left\{\sum_{i=j+3}^{\infty} \int_{a_{i-2}}^{a_{i}}|x| d F(x)+\int_{a_{0}}^{a_{j+1}}|x| d F(x)+\right. \\
& \left.\int_{0}^{a}|x+2| d F(x)-\int_{a_{1}}^{a_{j}}|x| d F(x)-\int_{a_{0}}^{a_{1}}|x| d F(x)-\int_{0}^{a_{j}^{j}}|x| d F(x)\right\}+ \\
& \left\{2 \sum _ { i = 1 } ^ { ( j - 1 ) / 2 } ( | a _ { 2 i } | - | a _ { 2 i - 1 } | ) \left(\sum_{i=j+3}^{\infty} \int_{a_{i-2}}^{a_{i}} d F(x)+\int_{a_{0}}^{a^{j+1}} d F(x)+\int_{a_{1}}^{a_{j}} d F(x)+\right.\right. \\
& \left.\left.\int_{0}^{a_{j}} d F(x)+\int_{0}^{a_{j+2}} d F(x)\right)\right\}+\left\{-2\left|a_{j}\right|\left(\sum_{i=j+3}^{\infty} \int_{a_{i-2}}^{a_{i}} d F(x)+\int_{a_{0}}^{a_{j+1}} d F(x)+\right.\right. \\
& \left.\left.\int_{0}^{a_{j}} d F(x)+\int_{0}^{a_{j+2}} d F(x)\right)\right\}+\left\{2\left|a_{j+1}\right|\left(\int_{0}^{a_{j}} d F(x)+\int_{0}^{a^{j+2}} d F(x)\right)\right\}+ \\
& \left\{2 \sum_{n=j+3}^{\infty} \sum_{i=j+1}^{n-1}\left|a_{i}\right| \int_{a_{n-2}}^{a_{n}} d F(x)\right\}=\left\{\left|a_{0}\right| \int_{c}^{d} d F(x)\right\}+\left\{\int_{c}^{d}|x| d F(x)-\right. \\
& \left.2 \int_{0}^{a_{0}}|x| d F(x)\right\}+\left\{2 \sum_{j=1}^{(j-1) / 2}\left(\left|a_{2 i}\right|-\left|a_{2 i-1}\right|\right)\left(1-\int_{a_{0}}^{a_{1}} d F(x)\right)\right\}+ \\
& \left\{-2\left|a_{j}\right|\left(1-\int_{a_{0}}^{a_{j}} d F(x)\right)\right\}+\left\{2\left|a_{j+1}\right| \int_{a_{j}}^{a_{j+2}} d F(x)\right\}+ \\
& \left\{2 \sum_{n=j+2}^{\infty} \sum_{i=j+1}^{n}\left|a_{i}\right| \int_{a_{n-1}}^{a_{n+1}} d F(x)\right\}
\end{aligned}
$$


But the sum of the last two terms $=2 \sum_{n=j+1}^{\infty} \sum_{i=j+1}^{n}\left|a_{i}\right| \int_{a_{n-1}}^{a_{n+1}} d F(x)$ $=2\left|a_{j+1}\right| \int_{a_{j}}^{a_{j+2}} d F(x)+2\left(\left|a_{j+1}\right|+\left|a_{j+2}\right|\right) \int_{a_{j+1}}^{a_{j+3}} d F(x)+$ $2\left(\left|a_{j+1}\right|+\left|a_{j+2}\right|+\left|a_{j+3}\right|\right) \int_{a_{j+2}}^{a_{j+4}} d F(x)+$ $2\left(\left|a_{j+1}\right|+\left|a_{j+2}\right|+\left|a_{j+3}\right|+\left|a_{j+4}\right|\right) \int_{a_{j+3}}^{a} d F(x)+\ldots$ $=2\left|a_{j+1}\right|\left(\int_{a_{j}}^{a_{j+2}} d F(x)+\int_{a_{j+1}}^{a_{j+3}} d F(x)+\int_{a_{j+2}}^{a_{j+4}} d F(x)+\ldots\right)+$ $2\left(\left|a_{j+2}\right|\left(\int_{a_{j+1}}^{a_{j+3}} d F(x)+\int_{a_{j+2}}^{a_{j+4}} d F(x)+\int_{a_{j+3}}^{a_{j+5}} d F(x)+\ldots\right)+\ldots\right.$ $=2\left|a_{j+1}\right|\left(1-\int_{a_{j}}^{a_{j+1}} d F(x)\right)+2\left|a_{j+2}\right|\left(1-\int_{a_{j+1}}^{a_{j+2}} d F(x)\right)+\ldots$ $=2 \sum_{i=j+1}^{\infty}\left|a_{i}\right|\left(1-\int_{a_{i-1}}^{a_{i}} d F(x)\right)$

$=2 \sum_{i=j+1}^{\infty}\left|a_{i}\right|\left\{1-\operatorname{sign}\left(a_{i}\right)\left[F\left(a_{i}\right)-F\left(a_{i-1}\right)\right]\right\}$

Since $\int_{C}^{d} d F(x)=1$ and $a_{0}, a_{j}$ have the same sign, the proof is complete. Q.E.D.

We have assumed that $J$ is a finite and nonempty set of odd (even) numbers in case (3) (case (4)). Owing to the nature of our assumptions, the number of elements in $J$ is not known. The following interesting result, however, will give us a high restriction on the number of elements that might belong to $J$. But let us first give the following definition. 
Definition 2.2: Let

$$
m_{k}=\inf \left\{D_{k}(a, F): a=\left\{a_{i} ; i \geq 0\right\} \varepsilon Q_{k}\right\} ; k=0,1,2,3 \text { and } 4 .
$$

If $a^{*}=\left\{a_{i}^{*}, i \geq 0\right\} \varepsilon_{Q_{k}}$ such that $m_{k}=D_{k}\left(a^{*}, F\right)$ then $a^{*}$ is said to be an optimal search path from class $Q_{k} ; k=0,1,2,3$ and 4 .

Theorem 2.3: Let $Q_{k}^{\prime}$ be a subclass of $Q_{k}(k=3$ or 4$)$ for which $J$ consists of only one element. If $a^{*}$ is an optimal search path from class $Q_{k}$, then $a^{*} \varepsilon Q_{k}^{\prime}$.

Proof: If $J$ consists of, at least, two elements, then for any search path $a=\left\{a_{i} ; i \geq 0\right\} \varepsilon Q_{k}(k=3$ or 4$)$, the search path $b=\left\{b_{i} ; i \geq 0\right\} \varepsilon_{k}$ defined by $b_{0}=a_{0}, b_{i}=a_{i+2}, i \geq 1$ has less expected cost than $a=\left\{a_{i} ; i \geq 0\right\}$. To see this, let $a_{0} \geq 0$ (the other case is dual), $\delta_{k}=\Delta_{k}(a, F)-\Delta_{k}(b, F)$ ( $k=3$ or 4$)$. If $k=3$, then by assumptions of the theorem and (2.13), elementary calculations yield:

$$
\delta_{3}=2\left(\left|a_{2}\right|-\left|a_{1}\right|\right)\left\{1+F\left(a_{1}\right)-F\left(a_{0}\right)\right\}+2 \sum_{i=1}^{(j-3) / 2}\left(\left|b_{2 i}\right|-\left|b_{2 i-1}\right|\right)\left[F\left(a_{1}\right)-F\left(b_{1}\right)\right]
$$

But, by hypothesis $b_{1} \leq a_{1},\left|a_{1}\right| \leq\left|a_{2}\right|$ and $j \geq 3$; hence $\delta_{3} \geq 0$. If $k=4$, then by (2.14) and assumptions of the theorem we similarly find;

$$
\delta_{4}=2\left(\left|a_{1}\right|-\left|a_{2}\right|\right)\left\{1+F\left(a_{0}\right)-F\left(a_{1}\right)\right\}+2 \sum_{i=1}^{(j-2) / 2}\left(\left|b_{2 i-1}\right|-\left|b_{2 i}\right|\right) \times\left[F\left(b_{1}\right)-F\left(a_{1}\right)\right]
$$

Since, by hypotheses $b_{1} \geq a_{1},\left|a_{1}\right| \geq\left|a_{2}\right|$ and $j \geq 4$, so $\delta_{4} \geq 0$. If $a \varepsilon Q_{3}$ and $j=3$ we are through. If, however, $j>3$ then for a search path $c=\left\{c_{i}, i \geq 0\right\} \varepsilon Q_{3}$ for which $c_{0}=b_{0}=a, c_{i}=b_{i+2}=a_{i+4}, i \geq 1$ we obtain $\delta_{3}=\Delta_{3}(b, F)-\Delta_{3}(c, F) \geq 0$. If we continue in the same manner, we shall eventually reach to a search path which satisfies the desired conclusion, because $J$ is a finite set. Similar argument holds for $\mathrm{arQ}_{4}$ and $j>4$.

Q.E.D.

The conclusions of theorem 2.3 imply that all optimal search paths from class $Q_{k}$ are contained in class $Q_{k}^{\prime}(k=3$ or 4$)$ where $Q_{3}^{\prime}$ consists of all search paths such that:

either

$$
\cdots \leq a_{5} \leq a_{3} \leq 0 \leq a_{1} \leq a_{0} \leq a_{2} \leq a_{4} \leq a_{6} \leq \ldots
$$

or

$$
\cdots \leq a_{6} \leq a_{4} \leq a_{2} \leq a_{0} \leq a_{1} \leq 0 \leq a_{3} \leq a_{5} \leq \ldots
$$

And $Q_{4}^{\prime}$ consists of all search paths such that 
either

$$
\cdots \leq a_{6} \leq a_{4} \leq 0 \leq a_{2} \leq a_{0} \leq a_{1} \leq a_{3} \leq a_{5} \leq \ldots
$$

or

$$
\cdots \leq a_{5} \leq a_{3} \leq a_{1} \leq a_{0} \leq a_{2} \leq 0 \leq a_{4} \leq a_{6} \leq \cdots
$$

We sha11, therefore, assume, from now on, that $Q_{k}$ is originally of the type $\ell_{k}^{\prime}$ i.e $\ell_{k} \equiv \ell_{k}^{\prime}$. Thus we obtain,

$$
\begin{aligned}
\Delta_{3}(a, F)= & -2\left|\int_{0}^{a_{0}}\right| x|d F(x)|+\left|a_{0}\right|-2\left|a_{1}\right|\left\{1+\operatorname{sign}\left(a_{0}\right)\left[F\left(a_{1}\right)-F\left(a_{0}\right)\right]\right\} \\
+ & 2 \sum_{i=2}^{\infty}\left|a_{j}\right|\left\{1-\operatorname{sign}\left(a_{i}\right)\left[F\left(a_{i}\right)-F\left(a_{i-1}\right)\right]\right\} \\
\Delta_{4}(a, F)= & -2\left|\int_{0}^{a_{0}}\right| x|d F(x)|-\left|a_{0}\right|+2\left|a_{1}\right|\left\{1-\operatorname{sign}\left(a_{0}\right)\left[F\left(a_{1}\right)-F\left(a_{0}\right)\right]\right\} \\
& -2\left|a_{2}\right|\left\{1-\operatorname{sign}\left(a_{0}\right)\left[F\left(a_{1}\right)-F\left(a_{2}\right)\right]\right\}+2 \sum_{i=3}^{\infty}\left|a_{i}\right|\left\{1-\operatorname{sign}\left(a_{i}\right) x\right. \\
& {\left.\left[F\left(a_{i}\right)-F\left(a_{i-1}\right)\right]\right\} }
\end{aligned}
$$

Remark 2.4: $\Delta_{k}(a, F)$ can be written in a common formula. Indeed, for $k=1,2$ :

$$
\begin{aligned}
\Delta_{k}(a, F)= & -2\left|\int_{0}^{a_{0}}\right| x\left|d F(x)-(-1)^{k}\right| a_{0}\left|+2 \sum_{i=1}^{\infty}\right| a_{i} \mid\left\{1-\operatorname{sign}\left(a_{i}\right) x\right. \\
& {\left.\left[F\left(a_{i}\right)-F\left(a_{i-1}\right)\right]\right\} }
\end{aligned}
$$

On the other hand, if we add $2\left|a_{1}\right|-2\left|a_{1}\right|$ to the right hand side of (2.20) and $2\left|a_{2}\right|-2\left|a_{2}\right|$ to the right hand side of $(2.21)$, then for $k=3,4$ we obtain:

$$
\begin{aligned}
\Delta_{k}(a, F)= & -2\left|\int_{0}^{a_{0}}\right| x|d F(x)|-(-1)^{k}\left|a_{0}\right|-4\left|a_{k-2}\right|+2 \sum_{i=1}^{\infty}\left|a_{i}\right|\left\{1-\operatorname{sign}\left(a_{i}\right) x\right. \\
& {\left.\left[F\left(a_{i}\right)-F\left(a_{i-1}\right)\right]\right\} }
\end{aligned}
$$

One can easily see from (2.22) and (2.23) that class $Q_{0}$ might be embedded in the other classes by taking $a_{0}=0$, because then $a_{k-2}=0$ for $k=3,4$.

Theorem 2.5: If $a=\left\{a_{i} ; i \geq 0\right\}$ is an (O.S.P.) from class $Q_{k}$, then $\left|a_{i+2}\right|>\left|a_{i}\right|$ for all $i \geq k-1 ; k=0,1,2,3 \& 4$. 
Proof: Suppose that the conclusion of the theorem fails, that is $\left|a_{m+2}\right|=\left|a_{m}\right|$ for some $m \geq k-1$. Define the search path $b=\left\{b_{i} ; i \geq 0\right\} \varepsilon Q_{k}$ by: $b_{i} \equiv a_{i}$ for $0 \leq i \leq m-1 ; b_{i}=a_{i+2}$ for $i \geq m$, then for $k=0,1,2,3 \& 4$ and $m \geq 1 ;(2.22)$ and (2.23) give:

$$
\begin{aligned}
\delta_{k}= & \Delta_{k}(a, F)-\Delta_{k}(b, F) \\
= & 2\left|a_{m}\right|\left\{1-\operatorname{sign}\left(a_{m}\right)\left[F\left(a_{m}\right)-F\left(a_{m-1}\right)\right]\right\}+2\left|a_{m+1}\right|\left\{1-\operatorname{sign}\left(a_{m+1}\right) x\right. \\
& {\left[F\left(a_{m+1}\right)-F\left(a_{m}\right)\right]+2\left|a_{m+2}\right|\left\{1-\operatorname{sign}\left(a_{m+2}\right)\left[F\left(a_{m+2}\right)-F\left(a_{m+1}\right)\right]\right\} } \\
& -2\left|a_{m+2}\right|\left\{1-\operatorname{sign}\left(a_{m+2}\right)\left[F\left(a_{m+2}\right)-F\left(a_{m-1}\right)\right]\right\} .
\end{aligned}
$$

Since $\operatorname{sign}\left(a_{m}\right)=\operatorname{sign}\left(a_{m+2}\right), \operatorname{sign}\left(a_{m+1}\right)=-\operatorname{sign}\left(a_{m}\right) \&\left|a_{m}\right|=\left|a_{m+2}\right|$, so

$$
\begin{aligned}
\delta_{k}= & 2\left|a_{m}\right|\left\{1-\operatorname{sign}\left(a_{m}\right)\left[F\left(a_{m}\right)-F\left(a_{m+1}\right)\right]\right\}+2\left|a_{m+1}\right|\left\{1-\operatorname{sign}\left(a_{m}\right) x\right. \\
& {\left.\left[F\left(a_{m}\right)-F\left(a_{m+1}\right)\right]\right\} } \\
= & 2\left(\left|a_{m}\right|+\left|a_{m+1}\right|\right)\left\{1-\operatorname{sign}\left(a_{m}\right)\left[F\left(a_{m}\right)-F\left(a_{m+1}\right)\right]\right\}
\end{aligned}
$$

Unless $\left|a_{m}\right|=\left|a_{m+1}\right|=0$, or $a_{m}=c, a_{m+1}=d$ or vice versa the differences $\delta_{k}>0$. But if $a_{m}=c, a_{l n+1}=d$ or vice versa, then $a_{m}, a_{m+1}$ are the last entries of the search path $\left\{a_{i} ; i \geq 0\right\}$ and no need to compare $\left|a_{m}\right|$ with $\left|a_{m+2}\right|$; because, then, $a_{m+2}$ does not exist. On the other hand, the condition $\left|a_{m}\right|=$ $\left|a_{m+1}\right|=0$ can not hold when $m \geq 1$ and $k \geq 1$, because either $\left|a_{m}\right| \geq\left|a_{0}\right|>0$ or $\left|a_{m+1}\right| \geq\left|a_{0}\right|>0$ (Recall the exclusion of the case $\left|a_{0}\right|=0$ for $k=1,2,3 \& 4$ and the fact that $m \geq 1$ for $k=2, m \geq 2$ for $k=3, m \geq 3$ for $k=4$ ). It remains to show that, such condition can not occur when $m=0$ for $k=1$ and when $m=-1,0$ for $k=0$. To see that $\left|a_{2}\right|>\left|a_{0}\right|$ for $k=1$. We assume contrary to the conclusion that $\left|a_{2}\right|=\left|a_{0}\right|$. Define $b=\left\{b_{i} ; i \geq 0\right\} \varepsilon Q_{1}, b_{0}=a_{0}$ and $b_{i}=a_{i+2}$ for $i \geq 1$ then

$$
\delta_{1}=\Delta_{1}(a, F)-\Delta_{1}(b, F)=2\left(\left|a_{1}\right|+\left|a_{2}\right|\right)\left\{1-\operatorname{sign}\left(a_{1}\right)\left[F\left(a_{1}\right)-F\left(a_{0}\right)\right]\right\}>0,
$$

because $\left|a_{2}\right| \geq\left|a_{0}\right|>0$ and $a_{1}, a_{0}$ are not the last entries as indicated above. The fact that $\left|a_{1}\right|>\left|a_{-1}\right|=0$ and $\left|a_{2}\right|>\left|a_{0}\right|=0$ has been shown by Beck [5] (see [5] theorem 6 ). Thus $\delta_{k}>0$ for all possibilities, which contradict our assumption that $a=\left\{a_{i} ; i \geq 0\right\}$ is an (O.S.P.) from class $Q_{k}$.

Remark 2.6: We have, so far, proved that if $a=\left\{a_{i} ; i \geq 0\right\}$ is an (O.S.P.) from class $Q_{k}$ then $\left|a_{i+2}\right|>\left|a_{i}\right|$ for a11 $i \geq k-1, k=0,1,2,3 \& 4$. Therefore, one can restrict his attention to such kind of search paths. But one should keep in mind that $\left|a_{i+2}\right| \geq\left|a_{i}\right|$ for $i<k-1$ when $k=1,2,3 \& 4$. The justifications for 
this might be, for instance, when $k=3$ and $x$ is between 0 and $a_{1}$, then $D_{3}(a, x)$ $=-|x|+\left|a_{0}\right|-2\left|a_{1}\right|+2\left|a_{2}\right|$. Thus for this special location of $x$, the reduction in $\left|a_{2}\right|$ reduces the travelled distance, hence the expected cost, which justifies the possibility that $\left|a_{2}\right|=\left|a_{0}\right|$. But the reduction in $\left|a_{1}\right|$ increases $D_{3}(a, x)$ which justifies taking $\left|a_{1}\right|>0$. Thus, in the special cases when the target $x$ lies between $a_{k-1}$ and $a_{k} ; k=1,2,3 \& 4$, it is reasonable to consider weak inequalities, between $\left|a_{i+2}\right|$ and $\left|a_{i}\right|$ for $i<k-1$, rather than strong ones. Therefore, in the "General case", the term "Strong search path" would mean: a search path $\left\{a_{i}, i \geq 0\right\}$ for which $\left|a_{i+2}\right|>\left|a_{i}\right|$ for $i \geq k-1 ; k=0,1,2,3$ \& 4 . Otherwise the search path is said to be weak.

\section{Existence of Optimal Search Paths in the General Case}

Besides the condition $A(F)<\infty$, authors in [5], [10] and [11] assumed certain conditions (necessary and sufficient), on the underlying distribution, under which, there exists a search path $a^{*}=\left\{a_{i}^{*} ; i \geq 0\right\}$ from class $Q_{0}$ such that $D_{0}\left(a^{*}, F\right)=m_{0}$. The techniques of those authors were to choose a search path $a^{(n)}=\left\{a_{i}^{(n)}\right\} \varepsilon_{Q_{0}}$ such that $\lim _{n \rightarrow \infty} D_{0}\left(a^{(n)}, F\right)=m_{0}$ (This choice is possible when $m_{0}<\infty$, which is equivalent to $A(F)<\infty$, by theorem 1.1). Then they proved that, for each $i,\left\{a_{i}^{(n)}\right\}$ is bounded in $n$, which precludes the possibility that $\underset{i}{a} \rightarrow \pm \infty$. The last result, then allows the possibility of getting a search path $\left\{a_{i}^{(n)}\right\}$ from class $Q_{0}$ such that $\lim _{n \rightarrow \infty} a_{i}^{(n)}=a_{i}^{*}$. Unless $a_{i}^{*} \equiv 0$ as a function of $i$, then it can be shown that $a^{*}=\left\{a_{i}^{*}\right\} \varepsilon_{0}$ and that $D_{0}\left(a^{*}, F\right)=m_{0}$. Thus $a^{*}=\left\{a_{i}^{*}\right\}$ is the desired search path. The heart of the work of Beck [5] and Franck [10] were lemma 3 in [5] and lemma 1 in [10], in which they give the necessary and sufficient conditions on $F$ so that $D_{0}\left(a^{*}, F\right)=m_{0}$ (condition (1.9) in [5] and (1.8) in [10]).

Therefore, we sha11, for the General Case, reprove, in detail, lemmas similar to lemma 3 of Beck [5] giving thus a modified sufficient conditions for the existence of an optimal search path in each of classes $Q_{k}$. The rest af the proofs, for the existence of an (O.S.P) in each $Q_{k}$, are not essential1y different from those of [5]. However, we shall include these proofs to show the modifications which suit the General Case.

Theorem 3.1: There exists a search path from class $Q_{k}(k=0,1,2,3 \& 4)$ with finite expected cost if and only if $A(F)<\infty$.

Proof: Suppose, first that $a=\left\{a_{i} ; i \geq 0\right\}_{\varepsilon Q_{k}}$ such that $D_{k}(a, F)<\infty$. 
It can be easily seen that for $k=1,2,3 \& 4$ (Theorem 1.1 has already dealt with the case $k=0)$, we either have $|x| \leq D_{k}(a, x)$ or $|x| \leq\left|a_{0}\right|$ or $|x| \leq D_{k}(a, x)+$ $\left|a_{0}\right|$. For simplicity we assume $|x| \leq D(a, x)+\left|a_{0}\right|$, so we obtain

$$
\begin{aligned}
A(F) & =\int_{C}^{d}|x| d F(x) \leq \int_{C}^{d} D_{k}(a, x) d F(x)+\int_{C}^{d}\left|a_{0}\right| d F(x) \\
& \left.=D_{k}(a, F)+\left|a_{0}\right|<\infty \text { (recall that }\left|a_{0}\right|<\infty\right)
\end{aligned}
$$

Conversely, let $A(F)<\infty$. Assume $c=-\infty, d=+\infty$ (the proof when one of $|c|,|d|$ is finite and the other is infinity can be done in a similar way, see for example, Balkhi [1] ch.1, Theorem 1). If we take the search path $a=\left\{a_{i}\right.$; $i \geq 0\} \varepsilon Q_{k}$, so that $a_{i}=(-2)^{i} \cdot \delta$ for $i \geq k-1 \quad(k=1,2,3 \& 4)$ where $\delta>0$ we have then $\left|a_{0}\right| \leq\left|a_{1}\right|=2 \delta$ for $k=2 ;\left|a_{1}\right| \leq\left|a_{0}\right| \leq\left|a_{2}\right|=2 \delta$ for $k=3$ and $\left|a_{2}\right| \leq$ $\left|a_{0}\right| \leq\left|a_{1}\right| \leq\left|a_{3}\right|=2 \delta$ for $k=4$. And then one can easily verify that

$$
D_{k}(a, x) \leq 9|x|+(k-1) \cdot \delta ; k=0,1,2,3 \& 4 .
$$

This gives:

$$
D_{k}(a, F) \leq 9 A(F)+(k-1) \cdot \delta<\infty
$$

The preceding theorem ensures that $A(F)<\infty$ is equivalent to $m_{k}<\infty$ for $k=0,1,2$, $3 \& 4$.

Lemma 3.2: If $F^{+}(0)<\infty$, then we can find a constant $M>0$ such that for any search path $a=\left\{a_{i} ; i \geq 0\right\}$ from class $e_{1}$ with $a_{2}>a_{0}>0 \geq a_{1}$ and $\left|a_{2}\right|+\left|a_{3}\right|$ $\leq M$, the search path $b=\left\{b_{i} ; i \geq 0\right\}$ defined by $b_{0}=a_{0} ; b_{i}=a_{i+2}$ for $i \geq 1$, is a search path from class $Q_{1}$ for which

$$
D_{1}(b, F) \leq D_{1}(a, F) \text {. }
$$

Proof: By the hypotheses of the lemma, it is clear that $b \varepsilon_{Q_{1}}$. Elementary calculations, then yield:

$$
\begin{aligned}
\delta_{1}=\Delta_{1}(a, F) & -\Delta_{1}(b, F)=2\left(\left|a_{2}\right|+\left|a_{1}\right|\right)\left\{1-\left[F\left(a_{0}\right)-F\left(a_{1}\right)\right]\right\} \\
& -2\left(\left|a_{2}\right|+\left|a_{3}\right|\right)\left[F\left(a_{2}\right)-F\left(a_{0}\right)\right] .
\end{aligned}
$$

Since $F^{+}(0)>\infty$, so we can find $M>0$, such that:$$
\left(1^{\circ}\right) \quad F\left(a_{0}\right)-F(-M)<(1 / 2) \operatorname{Pr}\left(x<a_{0}\right)
$$$$
\left(2^{\circ}\right) \quad\left(F(t)-F^{\prime}(0)\right) / t<1 / 2 M<\infty \forall 0<t<M
$$

Now: $0 \leq\left|a_{2}\right| \leq\left|a_{2}\right|+\left|a_{1}\right| \leq\left|a_{2}\right|+\left|a_{3}\right| \leq M$

$0 \leq\left|a_{1}\right| \leq\left|a_{2}\right|+\left|a_{1}\right| \leq M$ implies $a_{1}>-M$ which implies that 
$F\left(a_{1}\right) \geq F(-M)$

By $\left(1^{\circ}\right): 1 / 2<1-\left[F\left(a_{0}\right)-F(-M)\right] \leq 1-\left[F\left(a_{0}\right)-F\left(a_{1}\right)\right]$.

And by $\left(2^{\circ}\right):\left(F\left(a_{2}\right)-F\left(a_{0}\right)\right) / a_{2} \leq\left(F\left(a_{2}\right)-F(0)\right) / a_{2}<1 / 2 M$ which implies that $F\left(a_{2}\right)-F\left(a_{0}\right)<\left|a_{2}\right| \cdot 1 / 2 M$.

Hence:

$$
\delta_{1}>\left(2\left|a_{2}\right|\right)(1 / 2)-(2 M)\left(\left|a_{2}\right| \cdot 1 / 2 M\right)=0
$$

which in turn implies our conclusion.

Q.E.D.

Lemma 3.3: If $F^{-}(0)<\infty$ then we can find a constant $M>0$ such that for any search path $a=\left\{a_{i} ; i \geq 0\right\}$ from class $Q_{1}$ with $a_{2}<a_{0}<0 \leq a_{1}$ and $\left|a_{2}\right|+\left|a_{3}\right|$ $\leq M$ the search path $b=\left\{b_{i} ; i \geq 0\right\}$ defined by $b_{0}=a_{0}, b_{i}=a_{i+2} ; i \geq 1$ is a search path from class $Q_{1}$ for which

$$
D_{1}(b, F) \leq D_{1}(a, F)
$$

Proof: If we take $M$ to satisfy

$$
\begin{array}{ll}
\left(1^{\circ}\right) \quad F(M)-F\left(a_{0}\right)<(1 / 2) \operatorname{pr}\left(x>a_{0}\right) \\
\left(2^{\circ}\right) \quad(F(t)-F(0)) / t<1 / 2 M<\infty \quad \forall-M<t<0
\end{array}
$$

Then the rest of the proof is quite similar to that of the previous lemma.

Q.E.D.

Lemma 3.4: If $F^{-}(0)<\infty$ and $F^{+}(0)<\infty$, then we can find a constant $M>0$ such that for any search path from class $Q_{k} ; k=2,3,4$ with $\left|a_{k+1}\right|+\left|a_{k+2}\right| \leq M$, the search path $b=\left\{b_{i} ; i \geq 0\right\}$ defined by $b_{i}=a_{i}$ for $0 \leq i<k-1$ and $b_{i}=a_{i+2}$ for $i \geq k-1$ is a search path from class $Q_{k}$ for which

$$
D_{k}(b, F) \leq D_{k}(a, F) ; k=2,3 \& 4 \text {. }
$$

Proof: Let $a_{0}>0$, the other case is dua 1, and let $\delta_{k}=\Delta_{k}(a, F)-$ $\Delta_{k}(b, F)$. By the hypothesis of the lemma, simple calculations yie1d:

$$
\begin{aligned}
\delta_{2}= & 2\left(\left|a_{1}\right|+\left|a_{2}\right|\right)\left\{1-\left[F\left(a_{1}\right)-F\left(a_{0}\right)\right]\right\}-2\left(\left|a_{2}\right|+\left|a_{3}\right|\right)\left[F\left(a_{0}\right)-F\left(a_{2}\right)\right] \\
= & 2\left|a_{1}\right|\left\{1-\left[F\left(a_{1}\right)-F\left(a_{0}\right)\right]\right\}-2\left(\left|a_{2}\right|+\left|a_{3}\right|\right)\left[F\left(a_{0}\right)-F(0)\right] \\
& +2\left|a_{2}\right|\left\{1-\left[F\left(a_{1}\right)-F\left(a_{0}\right)\right]\right\}-2\left(\left|a_{2}\right|+\left|a_{3}\right|\right)\left[F(0)-F\left(a_{2}\right)\right] \\
\delta_{3}= & 2\left|a_{2}\right|\left\{1-\left[F\left(a_{2}\right)-F\left(a_{1}\right)\right]\right\}-2\left|a_{4}\right|\left[F\left(a_{1}\right)-F(0)\right] \\
& +2\left|a_{3}\right|\left\{1-\left[F\left(a_{2}\right)-F\left(a_{3}\right)\right]\right\}-2\left|a_{4}\right|\left[F(0)-F\left(a_{3}\right)\right]
\end{aligned}
$$


and

$$
\begin{aligned}
\delta_{4}= & 2\left|a_{3}\right|\left\{1-\left[F\left(a_{3}\right)-F\left(a_{4}\right)\right]\right\}-2\left|a_{5}\right|\left[F\left(a_{2}\right)-F(0)\right] \\
& +2\left|a_{4}\right|\left\{1-\left[F\left(a_{3}\right)-F\left(a_{4}\right)\right]\right\}-2\left|a_{5}\right|\left[F(0)-F\left(a_{4}\right)\right]
\end{aligned}
$$

Since both $F^{-}(0)$ and $F^{+}(0)$ are finite, so we can find $M>0$ so that:

$$
\begin{array}{ll}
\left(1^{\circ}\right) & F(M)-F\left(a_{0}\right)<(1 / 2) \operatorname{pr}\left(x>a_{0}\right) \\
\left(2^{\circ}\right) & (F(t)-F(0)) / t<1 / 2 M<\infty, \forall 0<|t|<M
\end{array}
$$

Now: for $k=2$ we have $0<a_{1}<M$, so $F\left(a_{1}\right) \leq F(M)$, which implies from $\left(1^{\circ}\right)$ :

$$
1-\left[F\left(a_{1}\right)-F\left(a_{0}\right)\right] \geq 1-\left[F(M)-F\left(a_{0}\right)\right] \geq 1 / 2 .
$$

By $\left(2^{\circ}\right)$ we have

$$
\frac{1}{2 M}>\frac{F\left(a_{1}\right)-F(0)}{a_{1}} \geq \frac{F\left(a_{0}\right)-F(0)}{\left|a_{1}\right|} \Longrightarrow F\left(a_{0}\right)-F(0)<\frac{1}{2 M} \cdot\left|a_{1}\right|
$$

On the other hand, since $\left|a_{2}\right|+\left|a_{3}\right| \leq\left|a_{3}\right|+\left|a_{4}\right| \leq M, 0 \geq a_{2}>-M$ so from (2०) we find

$$
\frac{1}{2 M}>\frac{F\left(a_{2}\right)-F(0)}{a}=\frac{F(0)-F\left(a_{2}\right)}{|a|} \Longrightarrow F(0)-F\left(a_{2}\right)<\frac{1}{2 M} \cdot\left|a_{2}\right| .
$$

Thus

$$
\delta_{2}>\left\{2\left|a_{1}\right|(1 / 2)-2 M(1 / 2 M) \cdot\left|a_{1}\right|\right\}+\left\{2\left|a_{2}\right|(1 / 2)-2 M \cdot(1 / 2 M) \cdot\left|a_{2}\right|\right\}=0+0=0 .
$$

By a suitable choice of $M$ in (3.3), and similar arguments to those of $k=2$ we can easily verify that $\delta_{3}>0$ and $\delta_{4}>0$.

Q.E.D.

Remark 3.5: It is to be noted that the condition $\left|a_{k+1}\right|+\left|a_{k+2}\right| \leq M$ and the two conditoins in (3.3) must be consistent. In fact, the inequality $\left|a_{k+1}\right|+\left|a_{k+2}\right| \leq M$ gives us a wide choice of $M$, but $\left(1^{\circ}\right)$ gives us an upper bound of this choice. On the other hand $\left(1^{\circ}\right)$ and $\left(2^{\circ}\right)$ are consistent since if we choose $M$ to satisfy $\left(2^{\circ}\right)$ then for all $0<t<M$ we have

$$
1 / 2 M>\frac{F(t)-F(0)}{t}>\frac{F(t)-F(0)}{M} \Longrightarrow F(t)-F(0)<1 / 2 .
$$

which is consistent with $\left(1^{\circ}\right)$. Similar argument can be done for $0>t>-M$.

Lemma 3.6: Let $a=\left\{a_{i} ; i \geq 0\right\}$ be a strong search path from class $Q_{k}$; $k=0,1,2,3$ and $4, \varepsilon>0$, and $M=M(F)$ defined as above.

(i) If $k=0$ or $k=1, F^{-}(0)<\infty$, then we can find a search path $b=\left\{b_{i} ; i \geq 0\right\}$ from class $Q_{k}(k=0$ or $k=1)$ such that 


$$
\begin{aligned}
& \left|b_{2}\right|+\left|b_{3}\right|>M(F), b_{0}=a_{0} \text { for } k=1 ; b_{1}>0 \text { for } k=0 \text { and } \\
& D_{k}(b, F)<D_{k}(a, F)+\varepsilon
\end{aligned}
$$

(ii) If $k=0$ or $k=1, F^{+}(0)<\infty$, then we can find a search path $b=\left\{b b_{i} ; i \geq 0\right\}$ from class $Q_{k}(k=0$ or $k=1)$ such that $b_{0}=a_{0}$ for $k=1, b_{1}<0$ for $k=0$, $\left|b_{2}\right|+\left|b_{3}\right|>M(F)$ and $D_{k}(b, F)<D_{k}(a, F)+\varepsilon$

(iii) If $k=2,3$ or $4, F^{-}(0)<\infty$ and $F^{+}(0)<\infty$, then we can find a search path $b=\left\{b_{i} ; i \geq 0\right\}$ from $Q_{k}$ so that $\left|b_{k+1}\right|+\left|b_{k+2}\right|>M(F)$ and $D_{k}(b, F)<D_{k}(a, F)$ $+\varepsilon$.

Proof: The proof for $k=0$ has been already shown by Beck [5]. For $k=1$, $2,3 \& 4$ we first show that for any search path $a=\left\{a_{i} ; i \geq 0\right\}$ from $Q_{k}$, there is a search path $e=\left\{e_{i} ; i \geq 0\right\} \varepsilon_{Q_{k}}$ with $D_{k}(e, F)<D_{k}\left(a, F^{\prime}\right)+\varepsilon$. To see this, we define $e=\left\{e_{i} ; i \geq 0\right\}$ as $e_{i} \equiv a_{i}$ for all $i$ except that $e_{5}$ be taken between $a_{3}$ and $a_{7}$ so that $0<\left|e_{5}\right|<\left|a_{5}\right|$ (Recall that in all cases we have $\left|a_{7}\right|>\left|a_{5}\right|$ $>\left|a_{3}\right|$ by theorem 2.5). Then by (2.22) and (2.23) we find:

$$
\begin{aligned}
\delta_{k}= & D_{k}(e, F)-D_{k}\left(a_{,} F\right)=2\left|e_{5}\right|\left\{1-\operatorname{sign}\left(e_{5}\right)\left[F\left(e_{5}\right)-F\left(a_{4}\right)\right]\right\} \\
+ & 2\left|e_{6}\right|\left\{1-\operatorname{sign}\left(e_{6}\right)\left[F\left(e_{6}\right)-F\left(e_{5}\right)\right]\right\}-2\left|a_{5}\right|\left\{1-\operatorname{sign}\left(a_{5}\right) \times\right. \\
& {\left.\left[F\left(a_{5}\right)-F\left(a_{4}\right)\right]\right\}-2\left|a_{6}\right|\left\{1-\operatorname{sign}\left(a_{6}\right)\left[F\left(a_{6}\right)-F\left(a_{5}\right)\right]\right\} }
\end{aligned}
$$

from which we find $\delta_{k}<2\left(\left|a_{5}\right|+\left|a_{6}\right|\right)\left|F\left(a_{5}\right)-F\left(e_{5}\right)\right|$. The right hand side of the last inequality can be made sufficiently small, say less than $\varepsilon>0$ by taking $e_{5}$ sufficiently near to $a_{5}$. Hence $D_{k}(e, F)<D_{k}(a, F)+\varepsilon$. Now if $\left|e_{k+1}\right|+\left|e_{k+2}\right|>M$ we are through. If not we take a new search path $d=\left\{d_{i} ; i \geq 0\right\}$ so that $d_{i}=e_{i}$ for $0 \leq i<k-1$ and $d_{i}=e_{i+2}$ for $i \geq k-1$ then by the previous three lemmas we have

$$
D_{k}(d, F) \leq D_{k}(e, F)<D_{k}(a, F)+\varepsilon
$$

If $\left|a_{k+1}\right|+\left|a_{k+2}\right|=\left|e_{k+3}\right|+\left|e_{k+4}\right|>M$ we are through. If not, then, since $M$ is bounded above, as can be noted from $\left(1^{\circ}\right)$ in $(3.2),(3.2)^{\prime}$ and (3.3), so if we continue the same process, we shall eventually reach to the desired search path i.e.

$$
D_{k}(b, F) \leq \ldots \leq D_{k}(d, F) \leq D_{k}(e, F)<D_{k}(a, F)+\varepsilon
$$

and $\left|b_{k+1}\right|+\left|b_{k+2}\right|>M$

Q.E.D. 
The result of the last lemma implies that; if $a^{(n)}=\left\{a_{i}^{(n)} ; i \geq 0\right\}$ is a search path from class $Q_{k}$ then there is a constant $M>0$ and a search path $b^{(n)}=$ $\left\{b_{i}^{(n)} ; i \geq 0\right\}$ based on $a^{(n)}$ as mentioned in the proof of the lemma such that $\left|b_{k+1}^{(n)}\right|+\left|b_{k+2}^{(n)}\right|>M$. This means that $b_{i}^{(n)}$ can not converge to zero as $n \rightarrow \infty$. Moreover for a search path $a{ }^{(n)}=\left\{a_{i}^{(n)} ; i \geq 0\right\} \varepsilon_{k}$, it is possible that $a_{i}^{(n)} \rightarrow$ $\pm \infty$. This circumstance, however, can not happen when $c=-\infty, d=+\infty$ as wị 11 be shown in the next lemma.

Lemma 3.7: If $c=-\infty, d=+\infty, a=\left\{a_{i} ; i \geq 0\right\} \varepsilon Q_{k} ; k=0,1,2,3$ and 4 for which $D_{k}(a, F)<2 m_{k}$, ther $a_{i}$ is bounded for each $i \geq 0$.

Proof: Let $p_{k}=\min \left\{\operatorname{pr}\left(x>\left|a_{k}\right|\right), \operatorname{pr}\left(x<-\left|a_{k}\right|\right)\right\}, k=0,1,2,3$ and 4 , then

$$
2\left|a_{k+1}\right| \cdot p_{k} \leq \int_{-\infty}^{\infty} 2\left|a_{k+2}\right| d F(x) \leq \int_{-\infty}^{\infty} D_{k}(a, x) d F(x)<2 m_{k} \Longrightarrow
$$

$\left|a_{k+2}\right|<m_{k} / p_{k+1}=b_{k+1}$ and so forth; if we assume that $p_{n}=\min \left\{p r\left(x>b_{n-1}\right)\right.$, $\left.\operatorname{pr}\left(x<-b_{n-1}\right)\right\}$, then $\left|a_{n+1}\right|<m_{k} / p_{n}=b_{n}$. Thus $a_{i}$ is bounded for a11 $i \geq k+1$. Since for $0 \leq i \leq k, a_{i}$ lies between $a_{k+1}$ and $a_{k+2}$, so $a_{i}$ is also bounded for those values of $i$.

Lemma 3.8: Let $M=M(F)$ defined as before. If $a=\left\{a_{i} ; i \geq 0\right\} \varepsilon Q_{k}$ such that $D_{k}(a, F)<2 m_{k},\left|a_{k+1}\right|+\left|a_{k+2}\right|>M(F)$, and $c<c^{-}<a_{0}<d^{+}<d$, then $a_{i} \varepsilon\left[c^{-}, a^{+}\right]$for at most $n_{0}$ values of $i$, where $n_{0}$ is constant.

Proof: Let $a_{0}>0, p=\min \left\{\operatorname{pr}\left(c^{-}<x<a_{k}\right), \operatorname{pr}\left(a_{k-1}<x<d^{+}\right)\right\}$, since $M<\left|a_{k+1}\right|+\left|a_{k+2}\right|<\left|a_{k+3}\right|+\left|a_{k+4}\right|<\ldots<\left|a_{2 n-1}\right|+\left|a_{2 n}\right|$, so for $c^{-}<a_{2 n} \leq a_{0}$ or $a_{0} \leq a_{2 n}<d^{+}$we have

$$
\begin{aligned}
2(n-1) M \cdot p & \leq \int_{C}^{d} 2(n-1) M \cdot p d F(x) \leq \int_{C}^{d} 2\left[\left|a_{k+1}\right|+\left|a_{k+2}\right|+\ldots+\left|a_{2 n-1}\right|+\left|a_{2 n}\right|\right] d F(x) \\
& \leq \int_{C}^{d} D_{k}(a, x) d F(x)=2 m_{k} \Longrightarrow n<\frac{m_{k}}{p M(F)}+1=\text { constant. }
\end{aligned}
$$

Thus the number of even entries between $C^{-}$and $a_{k}$ (or $a_{k-1}$ and $d^{+}$) is finite. A similar result holds for the odd entries. Hence the total number of entries between $C^{-}$and $d^{+}$is finite.

Q.E.D.

Theorem 3.9: Let $c=-\infty, d=+\infty$ and $A(F)<\infty$

(i) If $k=0$ or $1, F^{-}(0)<\infty$, then there exists an (O.S.P) $a^{*}=\left\{a_{i}^{*} ; i \geq 0\right\}$ from class $Q_{k}$ with $a_{1}^{*}>0$ for $k=0$ and $a_{0}^{*}<0$ for $k=1$

(ii) If $k=0$ or $1, F^{+}(0)<\infty$, then there exists an (O.S.P) $a^{*}=\left\{a^{*} ; i \geq 0\right\}$ from 
class $Q_{k}$ with $a_{1}^{*}<0$ for $k=0$ and $a_{0}^{*}>0$ for $k=1$

(iii) If $k=2,3$ or $4, F^{-}(0)<\infty$ and $F^{+}(0)<\infty$, then there exists an (0.S.P) from. $\operatorname{class} Q_{k}$.

Proof: Since $A(F)<\infty$, so $m_{k}<\infty$ for each $k=0,1,2,3 \& 4$ (Theorem 3.1 ). Therefore, for each $k=0,1,2,3 \& 4$, we can find a search path $a(n)=\left\{a_{i}^{(n)}\right.$, $i \geq 0\}$ from class $Q_{k}$ so that $D_{k}\left(a^{(n)}, F\right) \rightarrow m_{k}$ as $n \rightarrow \infty$. By lemma 3.6; for each $k$, there is a search path $b^{(n)}=\left\{b_{i}^{(n)} ; i \geq 0\right\}$, based on $a^{(n)}$ in the way mentioned in the proof of lemma 3.6 , such that

$$
\left|b_{k+1}^{(n)}\right|+\left|b_{k+2}^{(n)}\right| \geq M(F) \text { and } D_{k}\left(b^{(n)}, F\right)<D_{k}\left(a^{(n)}, F\right)+\delta_{n}
$$

where $\delta_{n} \rightarrow 0$ as $n \rightarrow \infty$. By lemma $3.7, b_{i}^{(n)}$ is bounded in $n$ for each $i$. So by the diagonal method we can find a subsearch path $b^{\left(n_{m}\right)}=\left\{b_{i}^{\left(n_{m}\right)} ; \ddot{i \geq 0}\right\}_{\varepsilon Q_{k}}$ so that $b_{i}^{\left(n_{m}\right)}$ convergent for each $i$ as $m \rightarrow \infty$. To avoid notational difficulties we assume that $b^{\left(n_{m}\right)}$ coincides with $b^{(n)}$. Now $\operatorname{let} a_{i}^{*}=\lim _{n \rightarrow \infty} b_{i}^{(n)} ; i \geq 0$, then it is clear that $a^{*}$ is a search path from class $Q_{k}$. Assume that $|c(n)|=$ $\max \left\{\left|a^{(n)}\right|,\left|a_{i}^{*}\right|\right\}$ for $i \geq k,\left|c_{i}^{(n)}\right|=\left|a_{i}^{(n)}\right|$ for $0 \leq i<k$ and that $c_{i}^{(n)}$ has the same sign as $a_{i}^{(n)}$, then $\left|c_{i}^{(n)}\right| \geq\left|a_{i}^{*}\right|$. Moreover, since $b_{i}^{(n)} \rightarrow a_{i}^{*}$, so $a_{i}^{(n)} \rightarrow$ $a_{i}^{*}$ which implies $c_{i}^{(n)} \rightarrow a_{i}^{*}$. Therefore we can find $\varepsilon_{1}>0$ and $n_{1}$ such that for a11 $i \geq 0$ we have $\left|a_{i}^{(n)}-a_{i}^{*}\right|<\varepsilon_{1}, \forall n>n_{1}$. Suppose that $x$ lies between, say $a_{2 m}^{*}$, $a_{2 m+1}^{*} ; m \geq k$. Since by the above arguments

$$
|| a_{i}^{(n)}|-| c_{i}^{(n)}|| \leq|| a_{i}^{(n)}|-| a_{i}^{*}|| \leq\left|a_{i}^{(n)}-a_{i}^{*}\right|<\varepsilon_{1}, \forall \cdot n>n_{1},
$$

so we have $\left|c_{i}^{(n)}\right| \leq\left|a_{i}^{(n)}\right|+\varepsilon_{1}$ for all $i \geq k, n>n_{1}$ and so

$$
\begin{aligned}
& D_{k}\left(c^{(n)}, x\right) \leq D_{k}\left(a^{(n)}, x\right)+4 m \cdot \varepsilon_{1}, \forall n>n_{1}\left(\text { For instance } D_{3}\left(c^{(n)}, x\right)=|x|+\right. \\
& \left|c_{0}^{(n)}\right|-2\left|c_{1}^{(n)}\right|+2 \sum_{i=2}^{2 m}\left|c_{i}^{(n)}\right| \leq|x|+\left|a_{0}^{(n)}\right|-2\left|a_{1}^{(n)}\right|+2 \sum_{i=1}^{2 m}\left(\left|a_{i}^{(n)}\right|+\varepsilon_{1}\right) \\
& \leq D_{3}\left(a{ }^{(n)}, x\right)+2 m \cdot 2 \varepsilon_{1} .
\end{aligned}
$$

As sume that $a_{2 m}^{*}<a_{2 m+1}^{*}$, then

$$
\begin{array}{r}
\int_{k}^{a_{2 m+1}^{*}} D_{k}\left(c^{(n)}, x\right) d F(x) \leq \int_{a_{2 m}^{*}}^{a_{2 m+1}^{*}} D_{k}\left(a^{(n)}, x\right) d F(x)+4\left(a_{2 m+1}^{*}-a_{2 m}^{*}\right) \varepsilon_{1} \cdot m \\
\forall n>n_{1} .
\end{array}
$$


But $c_{i}^{(n)} \rightarrow a_{i}^{*}$ implies that $D_{k}\left(c^{(n)}, x\right) \rightarrow D_{k}^{\prime}\left(a^{*}, x\right)$ for each $k$ and for any location of $x$. Therefore, we find $\varepsilon_{2}>0$ and $n_{2}$ such that

$$
\left|\int_{a_{2 m}^{*}}^{a^{*}} D_{k}(c), \int_{a_{2 m}^{*}}^{a_{2 m+1}^{*}} D_{k}\left(a^{*}, x\right) d F(x)\right|<\varepsilon_{2}, \forall n>n_{2} .
$$

Let $n_{0}=\max \left(n_{1}, n_{2}\right), \varepsilon=\max \left(\varepsilon_{1}, \varepsilon_{2}\right)$, then from the above arguments, for $n>n_{0}$, we have

$$
\begin{aligned}
& \int_{a_{2 m}^{*}}^{a_{2 m+1}^{*}} D_{k}\left(a^{*}, x\right) d F(x)<\int_{a_{2 m}^{*}}^{a_{2 m+1}^{*}} D_{k}\left(c^{(n)}, x\right) d F(x)+\varepsilon \leq \int_{a_{2 m}^{*}}^{a_{2 m+1}^{*}} D_{k}\left(a^{(n)}, x\right) d F(x) \\
& \quad+4 m \varepsilon\left(a_{2 m+1}^{*}-a_{2 m}^{*}\right)+\varepsilon \leq \int_{-\infty}^{\infty} D_{k}\left(a^{(n)}, x\right) d F(x)+4 m \varepsilon\left(a_{2 m+1}^{*}-a_{2 m}^{*}\right)+\varepsilon
\end{aligned}
$$

Taking the limits, as $n \rightarrow \infty$, of both sides we find

$$
\int_{a_{2 m}^{*}}^{a_{2 m+1}^{*}} D_{k}\left(a^{*}, x\right) d F(x) \leq D_{k}\left(a^{(n)}, F^{\prime}\right)+4 m \varepsilon\left(a_{2 m+1}^{*}-a_{2 m}^{*}\right)+\varepsilon \leq m_{k}
$$

From this we obtain: $\lim _{m \rightarrow \infty} \int_{a_{2 m}^{*}}^{a_{2 m+1}^{*}} D_{k}\left(a^{*}, x\right) d F(x) \leq m_{k}$ which in turn implies that $D_{k}\left(a^{*}, F\right) \leq m_{k}$. On the other hand $D_{k}\left(a^{*}, F\right) \geq m_{k}$ by the definition of $m_{k}$. Hence $D_{k}\left(a^{*}, F\right)=m_{k}$ which means that $a^{*}$ is an (O.S.P) from class $Q_{k}$.

Theorem 3.10: If $c>-\infty, d=+\infty$ and $A(F)<+\infty$, then theorem 3.9 holds.

Proof: Choose $a^{(n)}, b^{(n)}$ as in the preceding theorem. If $b_{i}^{(n)}$ is bounded in $n$ for each $i$, the proof of theorem 3.9 is valid for this theorem. Otherwise, let $m$ be the smallest $i$ for which $b_{i}^{(n)}$ is unbounded, i.e., $b_{i}^{(n)}$ is bounded in $n$ for each $0 \leq i<m$ but $b_{i}^{(n)}$ is unbounded in $n$ for $i \geq m$. As before; for $0 \leq i<m$ there is a subsearch path $\left\{b_{i} n_{j} ; i \geq 0\right\}$ such that $b_{i}\left(n_{j}\right)$ converges for each $0 \leq i<m$, but $b_{i} n_{j}$ diverges to $+\infty$ for $i 2 m$. For simplicity we assume that $\left\{b_{i}{ }^{j} ; i \geq 0\right\}$ coincides with $\left\{b_{i}^{(n)} ; i \geq 0\right\}$. As sume without loss of generality that $D_{k}\left(b^{(n)}, F\right) \leq 2 m_{k}$ then we have: 


$$
\left.2\left|b_{m}^{(n)}\right| p x\left\{x<b_{m-1}^{(n)}\right\}=\int_{C}^{b_{m-1}^{(n)}} 2\left|b_{m}^{(n)}\right| d F(x) \leq \int_{C}^{+\infty} D_{k}^{\left(b^{(n)}\right.}, x\right) d F(x) \leq 2 m_{k}
$$

which implies $\operatorname{pr}\left\{x<b_{m-1}^{(n)}\right\} \leq \frac{m_{k}}{b_{m}^{(n)}}$. Since $b^{(n)} \rightarrow \infty$ as $n \rightarrow \infty$, so

$\operatorname{pr}\left\{x<b_{m-1}^{(n)}\right\} \leq 0 \Longrightarrow \operatorname{pr}\left\{x<b_{m-1}^{(n)}\right\}=0$. Thus $b_{m-1}^{(n)} \rightarrow c$. As sume as before $a_{i}^{*}=\lim _{n \rightarrow \infty} b_{i}^{(n)}$, then $a^{*}=\left\{a_{0}^{*}, a_{1}^{*}, a_{2}, \ldots, a_{m-1}^{*},+\infty\right\}$ and as before $D_{k}\left(a^{*}, F\right)=$ $m_{k} \cdot$

Theorem 3.11: If $-\infty<C<a<d<+\infty$ and $A(F)<\infty$, then theorem 3.9 holds.

Proof: Define $a^{(n)}, b^{(n)}$ as in theorem 3.9. If $b_{i}^{(n)}$ is bounded in $n$ for each $i$ then the proof of theorem 3.9 can be applied here. Otherwise, we define $m$ as the least value of $i$ for which $b_{i}^{(n)}$ is unbounded. As in the previous theorem, one can easily show that $b_{m}^{(n)} \rightarrow a$. And if $a_{i}^{*}=\lim b_{i}^{(n)}$ for $0 \leq i<m$ then for the search path $a^{*}=\left\{a_{0}^{*}, a_{1}^{*}, \ldots, d, c\right\}$ or $a^{*}=\left\{a_{0}^{*}, a_{1}^{*}, \ldots, c, d\right\}$ we have $D_{k}\left(a^{*}, F\right)=m_{k}$.

Q.E.D.

Remark 3.12: We have indicated in theorem 3.9 that there exists an (o.s.P) from class $Q_{1}\left(Q_{0}\right)$ with $a_{0}^{*}<0\left(a_{1}^{*}>0\right)$ if $F^{-}(0)<+\infty$ and with $a_{0}^{*}>0 \quad\left(a_{1}^{*}<0\right)$ if $F^{+}(0)<+\infty$. But we did not refer to the sign of $a_{0}^{*}$ for $k=2,3$ or 4 . However we have in general to find an (0.S.P) from all search paths with $a_{0}>0\left(a_{1}>0\right.$ for class $\left.Q_{0}\right)$ and an (O.S.P.) from all search paths with $a_{0}<0 \quad\left(a_{1}<0\right.$ for class $Q_{0}$ ) and then choose the one with the least expected cost (e.g. see [1], table -3 and table -6 ).

\section{Some Applications of Linear Search}

As an application of linear search problems, beck [5] and Beck and Newman [7] have considered a man as an automobile searcher for another man who is located at same point of a certain road or highway. In addition to finding hidden particles on the real line Fristedt and Heath [11] have also applied linear search on some game problems. Some other applications of linear search may be cited:

(i) Search for a faulty unit in a large linear system such as: Petrol and gas supply lines (e.g. Algerian supply gas to Europe), many service systems 
like electrical power lines, telephone lines between cities or countries and some mining systems.

(ii) Search for an item of information stored in a memory (e.g. computer tapes).

(iii) Search for an enemy or a mine on a battlefront whose extension may be approximated by a straight line.

(iv) There are many kinds of search in which devices are used to detect targets or objects by finding their directions or azimuths (e.g. radar search). In cases when the target is located in a plane around the device, and the position of the target is given by the value of a random azimuth measured from a fixed azimuth (say zero azimuth). Then, one may start the search from some azimuth $a_{0}$ turn to the right (left) and to the left (right) until the target be detected. Restricting the support $[c, d]$ of the target's azimuth distribu= tion to the interval $[-\pi, \pi]$, we can, then easily show the equivalence between this search problem and the linear search problem. An illustration for case (1) is given in figure -2 .
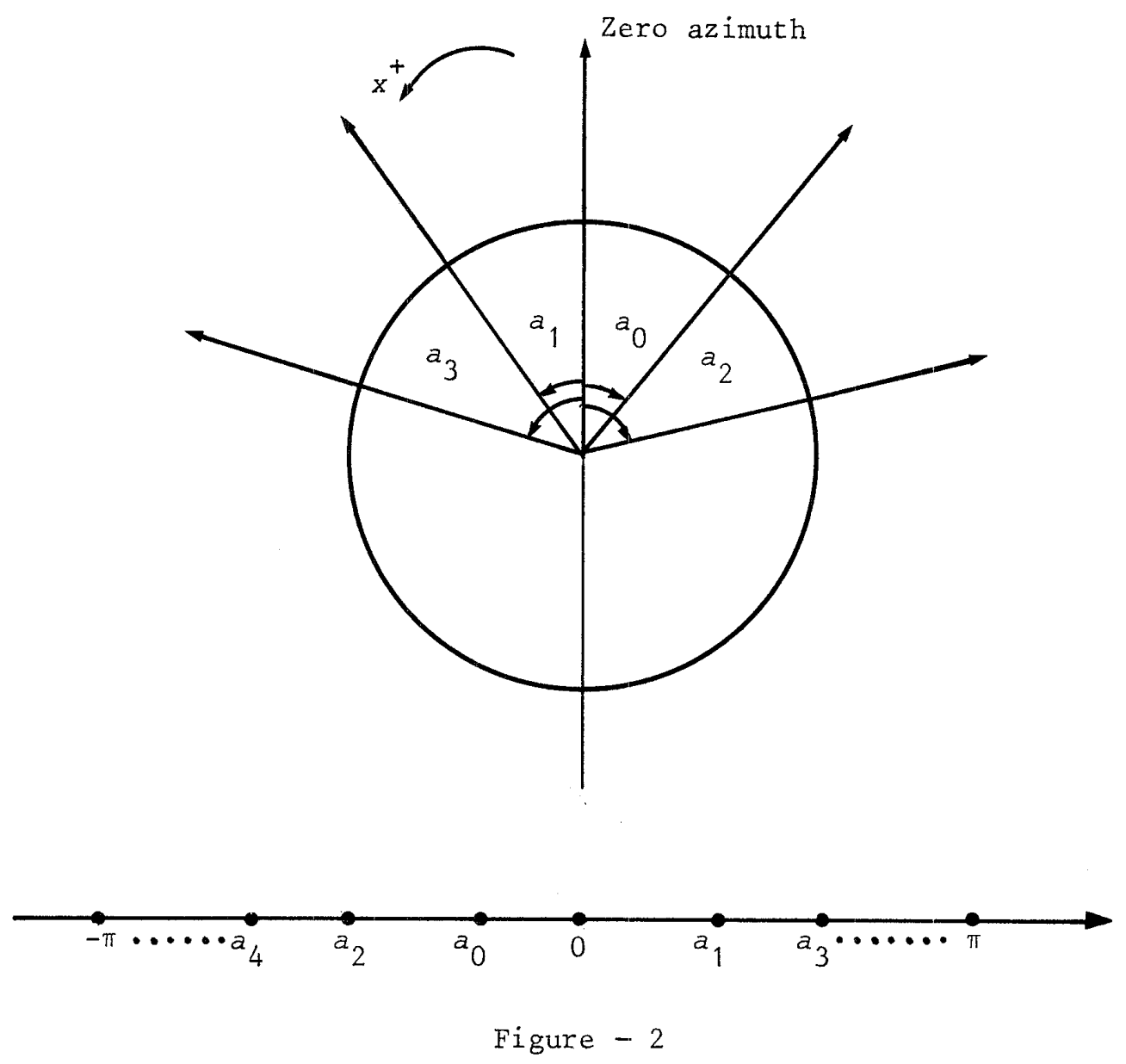
Moreover, it is to be mentioned that some more interesting applications may be found if this problem can be generalized to two or three dimentions. Some other types of various search problems, in which the target is located in the Euclidean $n$-space $(n \geq 1)$, or in one of a set of cel1s, can be found in Stone [14] and in many others in the literature.

\section{Acknowledgements}

This research is supported by the Research Center in the College of Science in King Saud University under the project No (Sat/1407/16). The author would like to express his thanks to the refrees for their helpful remarks.

\section{References}

[1] Balkhi, Z.: The Optimal Search Problem Theory and Computations, Ph.D. Thesis, 1983, Free University of Brusse1s.

[2] Bartle, R.: The Elements of Real Analysis, John Wiley and Sons, 1975.

[3] Bartle, R.: The Elements of Integration, John Wiley and Sons, 1966.

[4] Bauer, H.: Probability Theory and Elements of Measure Theory, 1971 , University of Erlangen-nurnberg (English translation, Rosenblatt, L.).

[5] Beck, A.: On the Linear Search Problem, Israel J. Math. 2 (1964), $221-228$.

[6] Beck, A.: More on the Linear Search Problem, Israel J. Math. 3 (1965), $61-70$.

[7] Beck, A. and Newman, D.: Yet more on the Linear Search Problem, Israel J. Math. 8 (1970), 419-429.

[8] Beck, A. and Warren, P.: The Return of the Linear Search Problem, Israel. J. Math. 14 (1973), 169-183.

[9] Feller, W.: An Introduction to Probability and Its Applications, John Wiley and Sons, 1970.

[10] Franck, W.: An Optimal Search Problem, SIAM Rev, 7, (1965), 503-512.

[11] Fristedt, B. and Heath, D.: Searching for a Particle on the Real Line, Adv. Appl. Prob. $6(1974), 79-102$.

[12] Johnson, N. and Kotz, S.: Continuous Univariate Distributions 1,2, Houghton Miffiin, Boston, MA, 1970.

[13] Rousseeuw, P.: Optimal Search Paths for Random Variables, J. Computational and Applied Mathematics, 9 (1983), 279-286. 
[14] Stone, L.: Theory of Optimal Search, Academic Press, New York, 1975.

Zaid T. BALKHI: Dept. of Statistics, College of Science, P.O.Box 2455

King Saud University, Riyadh-11451 Saudi Arabia 